Miscelánea 



\title{
Procesos recientes de reforma de la Administración Tributaria en el Perú y la necesidad de acometer uno nuevo*
}

\author{
Dante Mendoza Antonioli
}

Pagar tributos no es una actividad que la sociedad realice con especial dedicación. Para poder lograr el desarrollo de una conciencia tributaria en la población, el organismo encargado de la recaudación debe jugar un rol especial. Las personas perciben la importancia o no de pagar impuestos a través de los métodos y prácticas que ponga en ejecución el Estado. Así, si estos son flexibles o rígidos, el resultado en la percepción ciudadana variará y podrá ser determinante para el cumplimiento de las metas fiscales de corto y mediano plazo.

Una encuesta realizada a inicios de marzo de 2014 y publicada en el diario Perú21 el domingo 9 de marzo del mismo año ${ }^{1}$ (Datum Interna-

* Extracto de la investigación "Bases para la reforma de la Administración Tributaria", realizada bajo el auspicio del Instituto de Investigación Científica (IDIC), de la Universidad de Lima.

1 La encuesta fue realizada por la empresa Datum Internacional para Perú21 y Frecuencia Latina. Se la publicó como suplemento especial Pulso Perú el domingo 9 de marzo de 2014. Una muestra de los resultados se puede apreciar en el siguiente enlace: http://peru21.pe/economia/pulso-peru-81-cree-que-gobierno-gasta-inadecuadamente-tributos-2173387. La ficha técnica es la siguiente: encuesta realizada en Lima Metropolitana (no incluye Callao ni provincias) sobre 411 entrevistados entre el 28 de febrero y el 5 de marzo de 2014. Tiene un nivel de confianza de $95 \%$ y un margen de error de $+/-4,8 \%$. 
cional, 2014) arrojó como resultado que el 87 \% de los limeños considera que sí es importante el pagar impuestos. Sin embargo, esta percepción ciudadana pro pago de impuestos se ve afectada por el hecho de que el $64 \%$ piensa que el sistema tributario es complicado y el $81 \%$ cree que el Gobierno gasta inadecuadamente los impuestos que recauda.

La Sunat cumple un rol sumamente importante en conseguir que la población tenga una percepción más favorable, no solo de la necesidad de pagar impuestos, sino también de que esta actividad se convierta en una sencilla y, en el escenario ideal, automática. Del mismo modo, si bien es cierto que no es responsabilidad de esta institución el destino y/o calidad del gasto público, sí lo es el que la población la perciba de una manera justa y equilibrada.

\section{El contexto: Perú, $\mathbf{1 9 9 0}$}

El Perú de inicios de la década de 1990 era un país donde se podía apreciar la existencia de múltiples desequilibrios, tanto en el orden social y político como en el comercio internacional y las finanzas públicas. Fruto de decisiones políticas que no consideraron la reacción de la comunidad internacional, el país se encontraba no solo aislado del sistema financiero internacional y sin contar con reservas internacionales, sino que tampoco tenía la posibilidad de generarlas por sí mismo, sea por alguna industria desarrollada (que no existía) o por la explotación de algún recurso natural, actividad que se encontraba en manos de empresas públicas que llegaron a perder, entre 1989 y 1991, más de 5000 millones de soles (Paliza, 1999, pp. 17-18).

Hacia inicios de 1991, el déficit fiscal alcanzaba el $13 \%$ del PBI, en tanto que la presión tributaria no llegaba al 4,5\% del PBI, por lo que constituía una de las más bajas en el contexto internacional.

Una recaudación tributaria tan baja se explicaba no solo por el proceso de hiperinflación con que se inició la década, sino también por el colapso de un sistema tributario incoherente y absolutamente inmanejable desde la perspectiva de la administración, pues estaba compuesto por múltiples tributos, así como por una cantidad indeterminada de exenciones y privilegios fiscales. En adición, se debe tener en cuenta que, hacia junio de 1990, el sistema tributario era altamente dependiente del precio de los combustibles, del sector minero o del tipo de cambio. La recaudación estaba en función de lo que se pudiera cobrar en la venta 
de combustibles, lo cual nos convertía en vulnerables en extremo a cualquier vaivén de los precios internacionales del petróleo.

Este contexto se complementaba con una Administración Tributaria muy mal preparada, sobredimensionada y corrupta. El quinquenio previo había servido para generar el ingreso de miles de personas a la administración pública, sin cubrir requisito alguno distinto del de tener un carné del partido de gobierno. Estas personas vieron deteriorarse su capacidad adquisitiva de manera muy rápida, en razón de que la inflación avanzó mucho más rápido que cualquier sistema de actualización o indexación de ingresos aplicado en el sector público.

Un elemento adicional lo generaba el sector informal de la economía, que se había incrementado sensiblemente como resultado de la quiebra masiva de empresas formales operada a finales de la década previa. También fomentaba la informalidad el hecho de que esta resultaba una fuente segura de obtener ingresos, sin que el Estado sea capaz de evitar conductas empresariales al margen de la ley y, por supuesto, de la obligación de tributar.

Asimismo, la política de subsidios generalizados, vía tipos de cambio preferenciales, así como el cierre real de todos los mercados internacionales de capitales, generó que no sea posible mantener la caja fiscal con recursos distintos de la emisión inorgánica, con los conocidos resultados de hiperinflación.

Un país con esas características exigía un cambio dramático. En palabras de Guerra García (1995):

El vasto y complejo cuerpo legal que puso en marcha las reformas se orientó a liberalizar y desregular la economía, recomponiendo la relación entre los sectores públicos y privados, y revalorando el mercado y la competencia como elementos esenciales para la eficiente asignación de los recursos. (p. 80)

Tan pronto como un nuevo gobierno asumió el poder, en 1990, se puso en marcha un ambicioso programa de estabilización económica. La idea fue atacar las fuentes del desequilibrio financiero, con medidas poco populares pero efectivas, como eliminar los subsidios sustentados en el dólar MUC (mercado único de cambios). Este tipo de subsidios había destruido la economía nacional, a la vez que había servido de fuente inagotable de corruptelas en todo el Estado. Consistió en establecer un tipo de cambio artificial, denominado MUC, que sirviera de base 
para determinar si alguna actividad debería ser subsidiada o si debería más bien subsidiar a otras.

A partir de agosto de 1990, se inició un período en el cual las finanzas públicas fueron manejadas de manera ejemplar, bajo la atenta mirada y supervisión de la banca internacional. Se adoptó un sistema de flotación cambiaria y se unificó el mercado de divisas. Comenzó un proceso de consolidación del mercado como asignador de recursos y determinante de los precios en la economía, incluyendo el del dinero.

Como parte de las políticas de estabilización, se emprendió también un proceso de reinserción del Perú a la comunidad financiera internacional y la reducción de las tasas arancelarias (beneficiándose con claridad el mercado de importaciones). Igualmente, se implementó lo que el gobierno definió como "reformas estructurales", por las que se consolidó una posición gubernamental a favor del mercado y la libre competencia.

Uno de los elementos fundamentales de la reforma fue el de asegurar el incremento de ingresos tributarios en la línea de reducir, primero, y eliminar, después, el déficit fiscal del sector público. Para ello, a la par de aumentar la recaudación, se tomó una decisión que ha resultado ser fundamental en nuestra política macroeconómica: adaptar el gasto al ingreso. Todas estas acciones tuvieron un resultado positivo en cuanto al control de la inflación, logrando bajar la tasa de 7650 \% en 1990 a 139,2 \% en 1991.

\section{La reforma de la Administración Tributaria en la década de 1990}

A principios de la década, la Administración Tributaria aún estaba encargada al Ministerio de Economía y Finanzas. Si bien es cierto que en 1988 se promulgó la Ley 24829, que creó tanto la Superintendencia Nacional de Administración Tributaria (Sunat) como la Superintendencia Nacional de Aduanas (Aduanas), otorgándoles autonomía como organismos públicos descentralizados (OPD) del sector Economía y Finanzas, ello no se había verificado aún en la realidad. El cambio legislativo precedió al cambio real en la Administración Tributaria.

En efecto, el presupuesto de la primigenia Sunat estaba centralizado en el Ministerio de Economía y Finanzas y, por lo tanto, la ejecución del mismo tenía que pasar no solo por los filtros ministeriales, sino también por los procesos clásicos de la administración pública tradicional, caracterizados por ser extremadamente lentos y engorrosos. A inicios de la 
década de 1990, era bastante claro que mientras las decisiones en materia de gestión tengan que pasar por los procesos vigentes en los ministerios, resultaba imposible cumplir con cualquier objetivo de gobierno. El incremento excesivo y sin fundamento técnico alguno de trabajadores al servicio del Estado vino acompañado de medidas legislativas destinadas a justificar la presencia de estas personas, lo que complicaba y alargaba de manera absolutamente irracional e ineficiente el proceso de toma de decisiones en el sector público.

El gobierno de aquel entonces se propuso como un objetivo inmediato aumentar la recaudación a niveles que permitan equilibrar el presupuesto público. Para ello, tan importante como la Administración Tributaria era el tener un nuevo Código Tributario. Sin embargo, una Sunat dependiente del Ministerio de Economía no era adecuada para cumplir este objetivo. En esta primigenia Sunat, la corrupción era una compañera indesligable de la ineficiencia. Todo el personal de la nueva institución era el mismo de la Dirección General de Contribuciones, anterior órgano a cargo de la recaudación, del cual se ha dicho que era "un organismo muy desprestigiado $[. .$.$] , donde imperaba la coima$ como forma de resolver los asuntos tributarios de las empresas" (Zapata, 2005, p. 270). Por ello, más allá de un cambio de nombre, la Administración Tributaria no había pasado por ningún cambio sustantivo que permitiese cumplir con los objetivos gubernamentales de eficiencia y mejoras efectivas en la recaudación tributaria.

En este contexto, el primer elemento de la reforma que se debe tener en consideración es que el nuevo Código Tributario le otorgó facultades discrecionales muy grandes a la Sunat, sin imponer controles mínimos que permitan una adecuada defensa de los ciudadanos afectados por algún eventual exceso, y estableció presunciones que llegaron, incluso, a ser de iure, vale decir, que no admitían prueba en contrario.

Para acometer una verdadera reforma estructural de la Administración Tributaria se tuvo que pasar de la autonomía formal a la autonomía efectiva. De esta manera, se buscaba forjar una institución moderna y eficiente en sus objetivos, y establecer un sistema tributario equitativo y simple, en el que sea posible que la mayor parte de los ciudadanos (previstos en los supuestos de hecho generadores del tributo específico) tributen. Se creó una nueva institución "que contaba con trabajadores bien pagados y calificados, dotados tanto de instrumentos tecnológicos de primera línea como de una nueva moral" (Zapata, 2005, p. 271). 
De acuerdo con Estela (2000), para poder lograr este objetivo se contó con los siguientes elementos favorables:

a. Apoyo político desde el más alto nivel. Tanto el presidente de la República como el Congreso estaban alineados con el proceso de reforma.

b. Apoyo de las distintas fuerzas políticas representadas en el Congreso.

c. Apoyo de la opinión pública en general, la cual percibía que después de mucho tiempo el Estado se mostraba como capaz de combatir eficientemente los principales problemas del país de ese entonces, vale decir, la reducción de la inflación y la lucha contra el terrorismo. La gente comenzaba a percibir que el pago de impuestos no se iba solo al sueldo de corruptos e ineptos funcionarios, sino que también podía ser útil para que el Estado saque al país de la grave crisis por la que atravesaba.

d. El diseño y conducción de la reforma fue eminentemente técnico. Estuvo a cargo de los profesionales mejor calificados (y no de políticos). La mayoría provenía del Banco Central de Reserva (BCR), así como de la Ex Dirección General de Contribuciones (aquellos que tenían un prestigio y que no habían sido alcanzados por la corrupción generalizada). A ellos se sumaron técnicos enviados por algunos organismos internacionales como el Fondo Monetario Internacional (FMI), el Banco Interamericano de Desarrollo (BID) y el Centro Interamericano de Administradores Tributarios (CIAT).

Se partió por buscar reconstruir el sistema tributario nacional. El mecanismo utilizado fue el de la simplificación tributaria, que eliminó una gran cantidad de impuestos antitécnicos que resultaban de muy difícil y costosa recaudación. La presión tributaria, respecto del producto bruto interno, heredada al inicio del régimen era de tan solo $4,5 \%$ y resultaba claro para todos que mientras se mantuviera esa situación sería imposible que cualquier política económica tenga éxito.

Por otro lado, la neutralidad en la intervención del Estado en la economía jugó un rol importante en la consolidación del nuevo esquema de política económica. La tributación no fue la excepción en el modelo de un Estado neutral, y ello permitió que la Sunat pudiese actuar de manera uniforme en todos los sectores de la economía. La sensación de riesgo 
disminuyó al punto que, en los círculos empresariales, era común referirse a las obligaciones tributarias como parte natural de sus procesos productivos, y ya no como una situación excepcional en la que solo quienes tenían muy mala suerte y eran inspeccionados debían tributar.

La introducción de la Sunat en el escenario público generó un aumento visible de la eficiencia en la recaudación. Hacia 1997, ya se había logrado aumentar la presión tributaria a 14,1 \%, la cual, pese a que seguía entre las más bajas de la región, fue considerada un logro significativo. La opinión pública percibía con temor a la Sunat, pero era consciente de lo positivo de la presencia de esa institución en el andamiaje económico del país. La crisis de los años de 1980 se veía cada vez más como una pesadilla de la cual ya nos estábamos despertando.

La modernización de la administración tributaria vino de la mano con las nuevas medidas legales. Se eliminó la Libreta Tributaria, documento que no tenía más objeto que identificar a los contribuyentes con un número que no estaba vinculado a ningún dato histórico de aportes o contribuciones previas, el cual hubiera permitido contar con un insumo para el diseño de políticas tributarias o al menos como data para acciones de fiscalización. Su reemplazo, el RUC (Registro Único de Contribuyentes), era novedoso por ser virtual y tener utilidad únicamente ante la Sunat.

Se procedió también a introducir con mucha fuerza elementos de tecnología de última generación, que convirtieron a la Sunat en una de las administraciones tributarias más modernas de la región. Los instrumentos tecnológicos se orientaron a controlar la recaudación y el cumplimiento de las obligaciones tributarias.

La simplificación del sistema tributario nacional fue una de las acciones más significativas de esta primera etapa. Se logró en los primeros años revertir la dependencia del precio de los combustibles y generar que la mayor parte de la recaudación se concentre en dos impuestos: el Impuesto General a las Ventas y el Impuesto a la Renta. Este solo hecho explica que la recaudación sea estable y mucho menos vulnerable a cambios en los precios relativos o a cualquier variación sectorial interna o externa. El nuevo sistema tributario se basaba en cuatro grandes impuestos: Impuesto General a las Ventas (IGV), Impuesto Selectivo al Consumo (ISC), Impuesto a la Renta (IR) y el Impuesto a las Importaciones (IM). Por participación en la recaudación, el IGV era el más importante, seguido del IR, el ISC y, finalmente, el IM. 
Un gran avance en la época (y que hoy sigue siendo parte de la agenda pendiente) fue lograr una significativa ampliación de la base tributaria (contribuyentes que declaran y pagan sus obligaciones tributarias). Los diversos mecanismos de fiscalización, acompañados de la obligación para todos de emitir comprobantes de pago y de tener RUC para poder hacerlo, generaron que cada vez más y más personas, naturales y jurídicas, comiencen a pagar impuestos y sigan haciéndolo. Ello se debía a que los mecanismos de seguimiento se activaban una vez que uno hacía su primer pago o declaración. En un primer momento, la sola tenencia de un RUC importaba la necesidad de cumplir con obligaciones formales. En cambio, la anterior Libreta Tributaria era considerada por muchos como un documento más de identidad $\mathrm{y}$, por tanto, la tenían muchas personas que jamás habían tributado.

Cambiar la mentalidad de un país donde la adecuada identificación de un ciudadano requería de varios documentos fue una de las tareas en las que la administración tributaria colaboró eficazmente. La mayor parte de los trámites administrativos en los años ochenta debían ser cumplidos previa identificación con dos documentos de identidad. Usualmente, las personas contaban con tres: la libreta electoral, el brevete (licencia de conducir) y la libreta tributaria. Por ello, si una persona no sabía manejar, debía contar con la libreta tributaria; en caso contrario, no podía realizar algunos trámites administrativos. Con ello se desnaturalizaba la función de dicho documento, el cual no tenía ninguna utilidad para la administración tributaria, pues no brindaba data confiable ni siquiera de la condición efectiva de contribuyente de su portador.

Otro cambio importante de esta primera etapa lo constituyeron los regímenes tributarios especiales para el Impuesto General a las Ventas (Régimen Único Simplificado) y para el Impuesto a la Renta (Régimen Especial de Renta). En ambos casos, se permitía que los contribuyentes evitasen cálculos complejos contables para la determinación de sus obligaciones tributarias y se les exigía simplemente el pago de un porcentaje ciego de sus ventas o ingresos con carácter cancelatorio del impuesto a su cargo. Ello contribuyó considerablemente también a la ampliación de la base tributaria de contribuyentes efectivos.

Del mismo modo, también fue una novedad positiva la introducción del concepto de PRICO (principal contribuyente). De manera muy rápida, gracias a la guía de expertos del Banco Mundial, las autoridades de la Sunat se percataron de que un grupo muy reducido de contribuyen- 
tes explicaba el mayor porcentaje de ingresos tributarios. Ello provocó que se preparase una lista de "contribuyentes especiales", denominados PRICO (principales contribuyentes), a quienes se les aplicó un sistema informático especial de seguimiento diario respecto del cumplimiento de sus obligaciones tributarias. Así, a estos especiales grandes contribuyentes se les liquidaba y acotaba sus tributos en el día y con ello se podía obtener, además, reportes diarios de recaudación. Así pues, la Sunat llegó a tener una base de datos mucho más eficiente y confiable que la de la Dirección General de Presupuesto Público del Ministerio de Economía y Finanzas, órgano del Estado encargado del control y seguimiento de la planeación y ejecución del presupuesto de todas las entidades de la administración pública.

En la concepción de la Sunat y de quienes han estado vinculados a su quehacer, se considera que en esta primera etapa se lograron avances significativos en la creación de conciencia tributaria en la población. Sin embargo, más allá de continuar con los sorteos de comprobantes de pago (práctica tradicional de la anterior Dirección General de Contribuciones) y de intentar llegar a los colegios con charlas y campañas de difusión, lo real es que la cultura tributaria representa el gran déficit de todos los procesos de reforma operados hasta la actualidad. Las personas y empresas cumplen con la Sunat más por miedo que por estar convencidos de lo positivo que es tributar en nuestro país.

Transcurridos algo más de siete años de haberse iniciado el proceso de reformas, esta primera etapa llegó a su fin con resultados alentadores: cada vez más personas y empresas, que nunca antes habían pagado tributos, estaban tributando lo que les correspondía. La Administración Tributaria (Sunat) logró posicionarse en la opinión pública como una institución prestigiosa y confiable. Su personal era joven, de primer nivel, bien remunerado, muy poco corruptible (o incorruptible) y bastante motivado con el objetivo de lograr las metas de recaudación.

No obstante, al final de esta etapa era posible advertir, a partir del análisis de las variables base tributaria y recaudación, que el Perú evidenciaba una característica común a la mayoría de los países subdesarrollados: nuestro sistema se sostenía sobre una estructura piramidal, en la que menos del $1 \%$ de los contribuyentes explica algo más del $85 \%$ de la recaudación (Tagle, 2003). Con ello, los PRICO, y toda aquella gestión que de ellos se hiciese, adquirieron una fuerza e importancia tremenda en nuestras finanzas nacionales y, como se va a detallar 
más adelante, fue la puerta de entrada a la corrupción en la nueva administración tributaria.

\section{La contrarreforma y el regreso de la corrupción: 1997-2001}

Resulta importante el análisis de la evolución de la presión tributaria desde que todo lo avanzado durante la primera etapa (llegar al 14,1\%) comenzó a retroceder hasta que en el año 2001 solo alcanzó el 12,3 \% (véase el cuadro 1). Si bien se puede encontrar una explicación en la desaceleración del ciclo económico, no es menos cierto que también coincidió con una etapa en la que la política tributaria perdió importancia en la agenda pública (Murakami, 2012, p. 415).

Un indicador tan bajo explica la necesidad de seguir intentando procesos de reforma. La eficiencia en la recaudación debería ser un tema prioritario en la agenda política. Deben ser priorizadas adecuadamente acciones para ampliar la base tributaria, utilizando las herramientas que hoy brinda la tecnología, así como enfrentar el hecho de que se requiere eliminar las exoneraciones de carácter "político", que no hacen otra cosa que distorsionar la recaudación.

Respecto a este período, la comisión investigadora del Congreso indicó:

El aliento de la reforma duró dos años y se fue extinguiendo progresivamente, hasta que Jorge Baca Campodónico fue nombrado superintendente en 1997. En ese período y en los subsiguientes se habrían producido gruesas dificultades que comprometieron la gestión institucional. Los cambios de personal y organigrama fueron frecuentes y dieron grandes poderes a un grupo de funcionarios estables que podrían haber sido funcionales a la corrupción en curso [cursivas añadidas]. Al final del período, los cambios de superintendente fueron muy intensos llegando a ocupar el puesto por momentos muy reducidos. (Congreso de la República, 2002, p. 2)

El uso de la Sunat en esta etapa como mecanismo de presión y venganza política también fue observado por la propia comisión, que concluyó lo siguiente:

Es así que, de una manera similar al caso de la CGR, la Sunat habría servido para hostigar personas jurídicas y naturales vinculadas a la oposición. Si hacemos un balance, la Sunat parece repetir el patrón de conducta al que fueron sometidas las instituciones 
controladas por la mafia. Esto es, encubrió la corrupción en curso y promovió acusaciones contra los adversarios del gobierno. (Congreso de la República, 2002, p. 3)

\section{Cuadro 1}

Presión tributaria 1990-2015 (proyección)

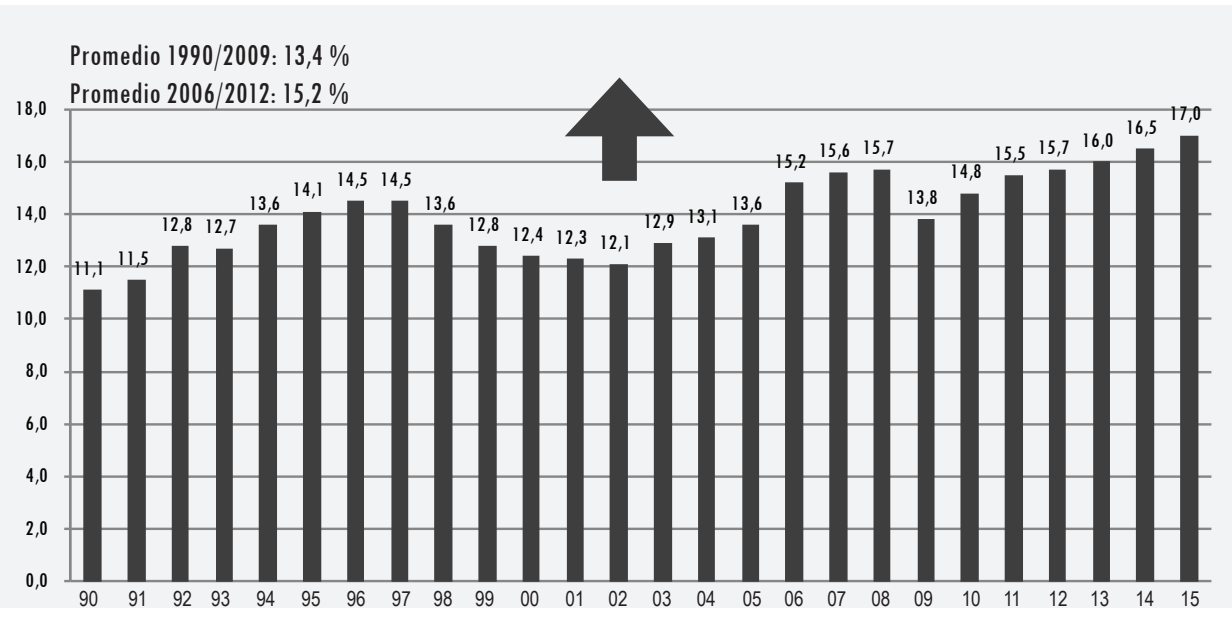

Fuente: Ministerio de Economía y Finanzas (2012, p. 1).

\subsection{El RUC sensible y el uso de la Sunat como medio de persecución política}

En 1997, se creó el RUC sensible. Para Zapata (2005):

[Esta medida estaba] orientada a proteger la secrecidad de los ingresos de Vladimiro Montesinos porque se había filtrado a la prensa una declaración jurada que lo revelaba como una persona que declaraba ingresos por más de US\$ 2 millones al año. De Montesinos, la coraza del RUC sensible se extendió a un grupo numeroso de altos funcionarios y siguió incrementándose hasta abarcar empresas, entre las que se contaban las registradas a nombre de Matilde Pinchi Pinchi. (p. 271) 
Según las declaraciones de Mauricio Muñoz-Nájar ${ }^{2}$ ante la Comisión Investigadora del Congreso, encargada de analizar los delitos económico-financieros perpetrados en el período 1990-200133, el RUC sensible se estableció porque la Sunat fue muy golpeada por la filtración al periodismo de la declaración jurada de Vladimiro Montesinos (Congreso de la República, 2002, p. 14). Cuando por los medios de prensa se conoció de los millonarios ingresos de Vladimiro Montesinos, el gobierno desarrolló el mecanismo del RUC sensible para, a través de la Sunat, proteger a las personas adeptas al régimen (dejando de fiscalizarlos), y controlar a quienes no lo eran. Nunca se publicó un reglamento que permita establecer mecanismos de transparencia para la incorporacióno desincorporación de contribuyentes a esa lista.

Muy de acuerdo con la política institucional de generar terror (tanto hacia sus propios trabajadores como con los contribuyentes):

El mecanismo consistía en un sistema de almacenamiento electrónico de información a la que se tenía acceso mediante claves preestablecidas que solo eran conocidas por el jefe de la Sunat y tres funcionarios de esa entidad. Las claves eran individuales, de tal forma que el sistema de control registraba el día, hora y funcionario que tenía acceso a la información tributaria protegida, a fin de identificar al responsable en caso de que se difunda [cursivas añadidas]. (Silva, 2001, p. 1)

Al no existir criterios claros y de obligatorio cumplimiento para la incorporación o desincorporación al régimen del RUC sensible, la decisión quedaba en manos del superintendente nacional. Con posterioridad, se detectó que muchas personas estuvieron incluidas en el mismo, sin estar enteradas de ello.

No obstante la magnitud de la falta a la confianza ciudadana en la custodia de su información tributaria, la investigación de auditoría interna, dispuesta durante el gobierno de transición (de noviembre de

2 Alto funcionario de la Sunat al momento de la ejecución de la política del RUC sensible.

3 Referente al proceso de privatización, el uso de recursos del Estado para el salvataje de empresas bancarias y no bancarias, licitaciones públicas, el uso de recursos públicos para conformar una red de corrupción, el uso de la Sunat y Aduanas como mecanismo de chantaje y presión, o de evasión tributaria y el mal uso de donaciones y otras ayudas, entre otros. 
2000 a julio de 2001), solo determinó responsabilidad en 12 personas: 4 exfuncionarios y 8 funcionarios. Ello evidencia que, antes que una práctica institucional e institucionalizada, la manipulación del RUC sensible fue el resultado de la debilidad institucional de la Sunat, fruto de las prácticas de gerencia poco transparentes y de terror al interior de los trabajadores de la organización.

Que 12 personas hayan podido tomar el control institucional y envolver a la Sunat en una práctica corrupta, sin que el resto lo advierta o tenga la capacidad real de oponerse, muestra que el modelo de organización y sus procesos internos debían ser replanteados. Es preocupante observar que, más de una década después, el modelo institucional sigue incólume, más allá de alguna modificación formal que se le pueda haber hecho en estos años.

El tema de la policía adscrita a la Sunat se convirtió en otro gran foco de corrupción. Creada con el fin de combatir la evasión, muy pronto “terminó envuelta en grandes actos de corrupción y extorsión de cientos de empresarios, convirtiendo la institución en un instrumento de protección de los amigos del régimen y de persecución a los opositores" (Zapata, 2005, p. 271). Este fue un período negro en la historia de la Sunat. "En este segundo período también se eliminaron los concursos como mecanismos de incorporación y ascensos internos, aumentando de modo dramático las decisiones arbitrarias adoptadas por criterios políticos y con total falta de transparencia" (Zapata, 2005, p. 271).

Pese a no ser un organismo cuya autoridad central cuente con estabilidad temporal en el cargo, puesto que "su jefe es nombrado por el presidente de la República que puede removerlo a voluntad en cualquier momento y sin necesidad de especificar causa alguna" (Zapata, 2005, p. 272), la Sunat de la primera etapa se las arregló para mantener cierta independencia y autonomía, de la mano del Ministerio de Economía y Finanzas. Sin embargo, hacia la segunda mitad de la década de 1990, el deterioro institucional era visible. La Sunat se convirtió en una herramienta más del gobierno para castigar a los opositores al régimen y beneficiar a una cúpula corrupta, dejando de fiscalizarlos y cobrarles impuestos.

Uno de los logros de la Sunat de la primera etapa había sido su profesionalismo y aparente independencia, lo que le había valido ganarse, si no la confianza ciudadana, por lo menos el miedo, basado en una imagen pública de incorruptibilidad. 


\section{Las medidas de la primera década de $\mathbf{2 0 0 0}$}

Esta década marca el declive institucional que se inicia con el descubrimiento de las redes de corrupción del gobierno anterior. Luego del gobierno de transición, se designó como superintendenta nacional a una persona que tenía la idea simplista de que toda la institución era corrupta, sin detenerse a analizar los procesos y las características que había observado la Sunat en aquella época. No se tomó en consideración que fue la debilidad del modelo institucional (que permitió que una camarilla tomase el poder y lo utilice con fines de corrupción y persecución política), y no la institución en sí misma, la causa de las prácticas corruptas. En un primer momento, se asumía que todos eran parte de la red de corrupción, mientras no se demuestre lo contrario.

Este hecho causó gran malestar en los funcionarios y empleados, quienes fueron sistemáticamente maltratados y a quienes se les fue retirando todos los beneficios laborales que habían obtenido a lo largo del tiempo. Las relaciones con los trabajadores fueron tan malas que se favoreció el surgimiento de una vigorosa y frondosa actuación sindical.

El estilo de maltrato al personal subsistió durante toda la década, con la excepción de un breve período en que estuvo a cargo la economista Graciela Ortiz, quien no venía ni de las canteras del Ministerio de Economía y Finanzas ni de la propia Sunat, e intentó reconstruir la confianza de los trabajadores en su propia institución. Durante toda la década, se mantuvo un perfil bastante bajo y se limitó a actuar siguiendo la inercia de recaudación y las metodologías de trabajo heredadas de los años noventa.

\subsection{El atraso tecnológico}

Un espacio aparte de reflexión lo constituye el hecho de que, pese a aparecer como improbable, en la Sunat lo real es el constante y endémico atraso tecnológico. Es una de las consecuencias de la mala gestión administrativa. La dirección de las entidades estatales resulta una actividad compleja y llena de caracteres propios que, por lo mismo, requiere de una gerencia profesional y especializada. La Sunat no la tiene. Se queda en tener expertos en tributación y aduanas, pero no en los sistemas administrativos y mucho menos en la dirección de entidades.

Los servidores de la Sunat han llegado a tener atrasos tecnológicos de más de diez años en la década pasada, por la sencilla razón de haber 
llevado mal los correspondientes procesos de licitación y/o concurso público. La consecuencia fue que estos tengan que ser declarados desiertos o, habiendo sido materia de impugnación, sean declarados nulos por el Tribunal del OSCE, ya sea porque se elaboraron mal los términos de referencia y se terminó solicitando cosas que el mercado no podía proveer, o porque se cometieron serias faltas administrativas durante los procesos.

Durante muchos años, los servicios de la plataforma virtual de la Sunat solo eran accesibles desde el navegador Internet Explorer. Como se puede apreciar en la imagen 1, hoy se ha avanzado a aceptar otros navegadores, pero aún se desconoce la existencia de los usuarios de los sistemas operativos que son utilizados por las computadoras Apple, o la de aquellos que se basan en Linux.

Hasta hace muy poco era común que los servidores de la Sunat se "cuelguen" los días de vencimiento de obligaciones tributarias y se nieguen a aceptar las declaraciones y transacciones de los contribuyentes. Lo irónico (y abusivo) de esta circunstancia es que a la institución le importaba muy poco el hecho de que la falta de cumplimiento de las obligaciones tributarias de los contribuyentes, muchas veces, fuera responsabilidad de la propia administración e, igual, terminaba imponiendo sanciones.

Aún hoy no es sencillo usar esta plataforma de servicios tecnológicos. El sistema devuelve constantes avisos de error, y presentar una simple declaración formal o intentar emitir un comprobante de pago electrónico puede convertirse en una actividad que demande mucho tiempo y paciencia por parte de los contribuyentes.

Los comprobantes de pago electrónicos, una de las grandes revoluciones en cuanto a administración tributaria se refiere, están listos para su implementación desde el año 2008. Sin embargo, solo el recibo por honorarios electrónico fue puesto en funcionamiento inicialmente. La factura electrónica tuvo que esperar hasta el año 2010 para poder ser utilizada por los contribuyentes y de manera parcial. Recién en 2013 se completó el proyecto con el acceso a las pequeñas y medianas empresas. 
Imagen 1

Compatibilidad de la plataforma virtual de la Sunat

\section{Compatibilidad}

SUNAT Operaciones en Línea - Oficina Virtual está optimizada para una resolución de $1.024 \times 768$ pixeles. Si dispone de un monitor con una resolución mayor, puede que el tamaño de letra resulte pequeño en algunos casos.

Los aplicativos de SUNAT Operaciones en Línea - Oficina Virtual se han probado en los navegadores MS Internet Explorer versión 8, Mozilla Firefox versión 26 y Google Chrome versión 32, o en versiones anteriores a los mismos. Para MS Internet Explorer versiones 9 y 10 debe activar la vista de compatibilidad de su navegador desde el menú "Herramientas".

Continuamos trabajando para la correcta visualización en otros navegadores.

\section{Los tiempos actuales: el régimen del terror tributario antes que el desarrollo de cultura tributaria o las viejas recetas de los noventa aplicadas al siglo XXI}

La percepción de la institución como una abusiva y que, en ocasiones, responde a intereses políticos antes que técnicos es común entre los contribuyentes. Así, se ha dicho:

La Sunat tiene una vara permisiva que yo llamaría la de la vista gorda. La aplica a muchos agricultores y comerciantes del sector agroindustrial, principalmente algodoneros, arroceros, azucareros y maiceros. ¿Por qué? No sé. Es evidente que la Sunat cuenta con todos los mecanismos tecnológicos y legales para formalizarlos [cursivas añadidas], pero -por alguna razón- no lo hace. La tercera vara es la abusiva e irracional. Aquella que, por errores realmente menores, y a veces por errores de la propia Sunat, aplica sanciones leoninas [cursivas añadidas] que pueden llegar al extremo de cerrar empresas serias y formales. Una sola vara, la del justo rigor. Esa es la única que debería tener la Sunat. (Cillóniz, 2013, p. 1)

No solo Cillóniz (2013) considera que la Sunat se ha politizado y que no es racional en su actuación. Economistas y estudiosos de los aspectos 
económicos públicos en el Perú, como Carlos Anderson, también lo consideran así. De este modo, al analizar los problemas de la Sunat, señala que cualquier cambio en la Administración Tributaria debe partir del respeto a los contribuyentes, al tiempo que destaca lo siguiente:

[...] la sociedad peruana ha ido legitimando la visión de que pagar impuestos es una cosa de tontos y no un acto de suprema responsabilidad. [...] La evasión tributaria y no el fútbol es el verdadero deporte nacional. [...] La verdadera tarea de los funcionarios de la Sunat no es solo aumentar la presión tributaria, sino recuperar la credibilidad de una institución que en algún momento llegó a ser materia de orgullo nacional. (Anderson, 2012, p. 1)

El conocimiento de los problemas subsistentes al interior del sistema tributario se evidenciaba ya en documentos internos, como la Memoria Institucional 2008, en la que se mencionaba:

No obstante estos logros, la evasión tributaria sigue siendo un mal endémico en nuestro país, toda vez que es un problema estructural, bastante generalizado en nuestra sociedad. Las causas son diversas y entre ellas se puede mencionar: la falta de conciencia tributaria, la complejidad de las normas tributarias [cursivas añadidas] y la estructura informal de nuestra economía. (Sunat, 2009, p. 3)

Sin embargo, la falta de gerencia de la administración tributaria y la escasa capacidad de propuesta para poder revertir esta situación, de manera inteligente y con pleno respeto a los derechos ciudadanos, es patente. Así, pese a haber advertido que dos de las tres principales causas de la evasión tributaria pueden ser superadas por una acción decidida y eficiente de la administración tributaria, la década concluyó sin que se hiciera mayor esfuerzo por revertirlas.

En efecto, la falta de conciencia tributaria y la complejidad de las normas tributarias constituyen dos causas de evasión tributaria contra las que la Sunat puede adoptar acciones directas y, no obstante, no lo hace. El tema de lo complejas que son no solo las normas tributarias, sino también la manera como la Sunat desarrolla sus acciones de fiscalización y cobro, es uno en el que la institución podría haber adoptado acciones más efectivas desde hace mucho tiempo.

Es cierto que la Sunat no tiene capacidad legislativa directa, pero no lo es menos que la mayor parte de las modificaciones legislativas en 
materia tributaria parten como iniciativa de esta institución. La corriente simplificadora de los años noventa ha quedado estéril en tiempos más recientes. Por el contrario, cada uno de los tributos vigentes tiende a volverse cada vez más complejo y de difícil entendimiento por el común de los contribuyentes. Cabe destacar que no solo se ha complejizado el aspecto sustantivo de los tributos, sino que no se ha adoptado una actitud decidida respecto a cambiar las metodologías clásicas de fiscalización y determinación de la deuda tributaria, que tantas críticas le han generado y le generan a la Sunat.

La historia reciente demuestra que no se ha aprendido en el proceso y aún se cree que, mientras más abusivo se sea, menor será la evasión tributaria. En febrero de 2012, se modificaron las normas tributarias ampliando las facultades discrecionales de la Sunat para determinar prácticas elusivas y sancionarlas drásticamente; se crearon nuevas limitaciones a los gastos deducibles de las empresas, en lo referente a inversiones, innovación y tecnología; y, finalmente, regresó, de manera discreta pero cierta, la obligación de pagar primero y reclamar después (a través de la obligación de garantizar el $100 \%$ de la deuda impugnada, como requisito para interponer una demanda judicial en contra de las decisiones que adopta, en última instancia administrativa, el Tribunal Fiscal). Todas estas medidas, lejos de fortalecer el compromiso de las personas con el pago de sus tributos, no han hecho otra cosa que mellar aún más la confianza en la institucionalidad tributaria.

En el período de gobierno 2011-2016, la Sunat ha desplegado una estrategia netamente de cobrador, sin detenerse a evaluar las características de sus métodos y su compatibilidad con los principios más elementales del derecho, como lo son los de razonabilidad, proporcionalidad, presunción de licitud y debido procedimiento. Se siguen aplicando en 2016 las mismas acciones que son percibidas como abusivas por la población y cuya compatibilidad con los derechos fundamentales reconocidos en la Constitución resulta más que discutible. Así, se cierran locales sin que haya concluido el procedimiento; se utilizan cartelones indignos para "informar" a la población del cierre (los sambenitos del siglo XXI); las comisiones de auditoría a las empresas se instalan permanentemente en las oficinas de los contribuyentes (a costa de ellas); se presumen infracciones tributarias sin haber cruzado adecuadamente la información disponible; se incide en el cumplimiento de aspectos formales que ya no tienen sentido en la actualidad (el avan- 
ce tecnológico las vuelve obsoletas); se infringe claramente el principio de legalidad en materia tributaria, al perseguir la elusión como si fuera evasión; entre otras prácticas.

La soberbia institucional llega al extremo de interponer acciones judiciales en contra de lo resuelto por el Tribunal Fiscal, máxima instancia administrativa en el procedimiento tributario. Una acción de esta naturaleza es percibida como de "persecución" por el común de las personas. Es muy complicado sustentar y defender la posición de una institución que ni siquiera ha entendido su ubicación como órgano de primera instancia en el espectro de la administración pública.

En adición, las interpretaciones en contra de los contribuyentes que realiza la administración tributaria no se limitan a temas de temporalidad en la determinación del derecho aplicable, sino que se extienden, en general, a todas y cada una de sus decisiones. Así, se coloca a los contribuyentes en una patente situación de indefensión que no puede ser quebrada, ni siquiera por la intervención directa del Poder Judicial, desde que se exige afianzar el 100 \% de la obligación en disputa como requisito para acceder a la tutela judicial. Este tipo de comportamiento es el que genera una percepción ciudadana muy poco amable para con la Sunat, la que es vista, hasta hoy, como una entidad eminentemente abusiva y de la que hay que cuidarse y, en la medida de lo posible, evitarla.

Por ello, la necesidad de intentar un nuevo proceso de reforma de la Administración Tributaria, en el que se parta de lo elemental: la persona. Si la institución comienza por respetar a sus trabajadores y a los contribuyentes, se habrán sentado las bases para construir y desarrollar una cultura tributaria que nos beneficie a todos y permita construir un mejor futuro para el país.

\section{Referencias}

Anderson, C. (10 de febrero de 2012). La Sunat y sus excesos. Gestión. Recuperado de http://gestion.pe/impresa/noticia/sunat-sus-excesos/2012-02-10/44500

Cillóniz, F. (27 de febrero de 2013). Las tres varas de la Sunat. Perú21. Recuperadodehttp://peru21.pe/impresa/tres-varas-sunat-2119265

Congreso de la República. (27 de mayo de 2002). Acta de la sesión reservada de la Comisión Investigadora del Congreso de los Delitos, 
encargada de analizar los delitos económico-financieros perpetrados en el período 1990-2001 en el proceso de privatización, el uso de recursos del Estado para el salvataje de empresas bancarias y no bancarias, licitaciones públicas, el uso de recursos públicos para conformar una red de corrupción, el uso de la Sunat y Aduanas como mecanismo de chantaje y presión o de evasión tributaria y el mal uso de donaciones y otras ayudas recibidas, entre otros. Recuperado de http://www.congreso.gob.pe/comisiones/2002/ CIDEF/transcri/sunat_najar.pdf

Datum Internacional. (9 de marzo de 2014). Encuesta Pulso Perú. Perú21. Recuperado de http://peru21.pe/economia/pulso-peru-81-cree-que-gobierno-gasta-inadecuadamente-tributos- 2173387

Estela, M. (2000). Fortaleciendo la honestidad en la administración de impuestos: el caso de la Sunat en el Perú. Moneda, Revista del BCR, 123, 37-42.

Guerra García, F. (1995). La Reforma del Estado. Socialismo y Participación, 69, 80.

Ley 24829. (31 de mayo de 1988). Recuperado de http://www.sunat.gob. pe/legislacion/sunat/ley-24829.pdf

Ministerio de Economía y Finanzas. (2012). Propuestas de modificación al sistema tributario. Recuperado de http://www.mef.gob.pe/contenidos/comun_notp/presentaci/2012/present_sist_tributario 23052012.pdf

Murakami, Y. (2012). Perú en la era del Chino. La política no institucionalizada y el pueblo en busca de un salvador. Lima, Perú: Instituto de Estudios Peruanos.

Paliza, R. (1999). Impacto de las privatizaciones en el Perú. Revista de Estudios Económicos. Banco Central de Reserva, 4, 9-37.

Silva, V. (1 de marzo de 2001). Montesinos también controló la Sunat: el RUC sensible fue uno de sus mecanismos. La República. Recuperado de http://www.larepublica.pe/01-03-2001/montesinos-tambien -controlo-la-sunat-el-ruc-sensible-fue-uno-de-sus-mecanismos

Superintendencia Nacional de Aduanas y de Administración Tributaria (Sunat). (2009). Memoria Institucional 2008. Lima, Perú: Autor. 
Tagle, M. del C. (2003). El ciclo económico y la recaudación tributaria en el Perú: 1993-2001. (Tesis). Universidad Nacional San Antonio Abad del Cusco, Perú.

Zapata, A. (2005). La corrupción en el fujimorismo. En F. Portocarrero (Ed.). El pacto infame: estudios sobre la corrupción en el Perú (pp. 247-285). Lima, Perú: Red para el Desarrollo de las Ciencias Sociales en el Perú. 
